\title{
Topological Properties of Real Normed Space ${ }^{1}$
}

\author{
Kazuhisa Nakasho \\ Shinshu University \\ Nagano, Japan
}

\author{
Yuichi Futa \\ Japan Advanced Institute \\ of Science and Technology \\ Ishikawa, Japan
}

\author{
Yasunari Shidama \\ Shinshu University \\ Nagano, Japan
}

\begin{abstract}
Summary. In this article, we formalize topological properties of real normed spaces. In the first part, open and closed, density, separability and sequence and its convergence are discussed. Then we argue properties of real normed subspace. Then we discuss linear functions between real normed speces. Several kinds of subspaces induced by linear functions such as kernel, image and inverse image are considered here. The fact that Lipschitz continuity operators preserve convergence of sequences is also refered here. Then we argue the condition when real normed subspaces become Banach's spaces. We also formalize quotient vector space. In the last session, we argue the properties of the closure of real normed space. These formalizations are based on [19](p.3-41), 2] and [34](p.3-67).
\end{abstract}

MSC: 46B20 46A19 03B35

Keywords: functional analysis; normed linear space; topological vector space MML identifier: NORMSP_3, version: 8.1.03 5.25.1220

The notation and terminology used in this paper have been introduced in the following articles: [3], 23], [14, [4, [5], [20], 25], [15], [18, , 9], 11, [6], [17], [31], [32], 26], [27], 28], 8], [22], [11, 16], [30], 10], 33], [12], 13], 29], and [7].

\footnotetext{
${ }^{1}$ This work was supported by JSPS KAKENHI 22300285.
} 


\section{Open And Closed}

Let $X$ be a real normed space. One can check that there exists a subset of $X$ which is open and closed.

Now we state the proposition:

(1) Let us consider a real normed space $X$ and a subset $R$ of $X$. Then $R$ is closed if and only if $R^{\mathrm{c}}$ is open.

Let $X$ be a real normed space and $R$ be a closed subset of $X$. Let us observe that $R^{\mathrm{c}}$ is open.

Now we state the proposition:

(2) Let us consider a real normed space $X$ and a subset $R$ of $X$. Then $R$ is open if and only if $R^{\mathrm{c}}$ is closed.

Let $X$ be a real normed space and $R$ be an open subset of $X$. Let us observe that $R^{\mathrm{c}}$ is closed and $\Omega_{X}$ is closed and $\emptyset_{X}$ is closed and $\Omega_{X}$ is open and $\emptyset_{X}$ is open.

Let $P, Q$ be closed subsets of $X$. Note that $P \cap Q$ is closed as a subset of $X$ and $P \cup Q$ is closed as a subset of $X$.

Let $P, Q$ be open subsets of $X$. Let us observe that $P \cap Q$ is open as a subset of $X$ and $P \cup Q$ is open as a subset of $X$.

Let $Y$ be a subset of $X$. The functor $\bar{Y}$ yielding a subset of $X$ is defined by

(Def. 1) there exists a subset $Z$ of LinearTopSpaceNorm $X$ such that $Z=Y$ and it $=\bar{Z}$.

One can verify that $\bar{Y}$ is closed.

Now we state the propositions:

(3) Let us consider a real normed space $X$, a subset $Y$ of $X$, and a subset $Z$ of LinearTopSpaceNorm $X$. If $Y=Z$, then $\bar{Y}=\bar{Z}$.

(4) Let us consider a real normed space $X$ and a subset $Z$ of $X$. Then $Z \subseteq \bar{Z}$. The theorem is a consequence of $(3)$.

Let us consider a real normed space $X$, a subset $Y$ of $X$, and an object $v$. Now we state the propositions:

(5) If $v \in$ the carrier of $X$, then $v \in \bar{Y}$ iff for every subset $G$ of $X$ such that $G$ is open and $v \in G$ holds $G$ meets $Y$.

Proof: Reconsider $Z=Y$ as a subset of LinearTopSpaceNorm $X$. For every subset $G_{0}$ of LinearTopSpaceNorm $X$ such that $G_{0}$ is open and $v \in$ $G_{0}$ holds $G_{0}$ meets $Z$ by [9, (33)].

(6) $v \in \bar{Y}$ if and only if there exists a sequence $s_{2}$ of $X$ such that $\operatorname{rng} s_{2} \subseteq Y$ and $s_{2}$ is convergent and $\lim s_{2}=v$. 
Proof: Reconsider $Z=Y$ as a subset of LinearTopSpaceNorm $X . \bar{Z}=\bar{Y}$. For every subset $G$ of LinearTopSpaceNorm $X$ such that $G$ is open and $v \in G$ holds $G$ meets $Z$ by [9, (22)], [18, (7)], [5, (4)].

(7) Let us consider a real normed space $X$ and a subset $A$ of $X$. Then there exists a family $F$ of subsets of $X$ such that

(i) for every subset $C$ of $X, C \in F$ iff $C$ is closed and $A \subseteq C$, and

(ii) $\bar{A}=\bigcap F$.

Proof: Reconsider $B=A$ as a subset of LinearTopSpaceNorm $X$. Consider $G$ being a family of subsets of LinearTopSpaceNorm $X$ such that for every subset $C$ of LinearTopSpaceNorm $X, C \in G$ iff $C$ is closed and $B \subseteq C$ and $\bar{B}=\bigcap G$. Reconsider $F=G$ as a family of subsets of $X$. For every subset $C$ of $X, C \in F$ iff $C$ is closed and $A \subseteq C$ by [9, (32)].

Let us consider a real normed space $X$ and subsets $A, B$ of $X$. Now we state the propositions:

(8) If $A \subseteq B$, then $\bar{A} \subseteq \bar{B}$. The theorem is a consequence of (3).

(9) $\overline{A \cup B}=\bar{A} \cup \bar{B}$. The theorem is a consequence of (3).

(10) $\overline{A \cap B} \subseteq \bar{A} \cap \bar{B}$. The theorem is a consequence of (3).

Let us consider a real normed space $X$ and a subset $A$ of $X$. Now we state the propositions:

(11) $A$ is closed if and only if $\bar{A}=A$. The theorem is a consequence of (3).

(12) $A$ is open if and only if $\overline{\Omega_{X} \backslash A}=\Omega_{X} \backslash A$. The theorem is a consequence of $(3)$.

(13) Let us consider a real normed space $X$, a subspace $Y$ of $X$, and a subset $C_{3}$ of $X$. Suppose $C_{3}=$ the carrier of $Y$. Then $\overline{C_{3}}$ is linearly closed.

Proof: For every points $v, u$ of $X$ such that $v, u \in \overline{C_{3}}$ holds $v+u \in \overline{C_{3}}$ by (6), [5, (11), (4)], [26, (20)]. For every real number $r$ and for every point $v$ of $X$ such that $v \in \overline{C_{3}}$ holds $r \cdot v \in \overline{C_{3}}$ by (6), [18, (22), (28)], [5, (11), $(4)]$.

\section{Density}

Let $X$ be a real normed space and $A$ be a subset of $X$. We say that $A$ is dense if and only if

(Def. 2) $\bar{A}=\Omega_{X}$.

One can check that $\Omega_{X}$ is dense and there exists a subset of $X$ which is open, closed, and dense.

Now we state the propositions: 
(14) Let us consider a real normed space $X$ and a subset $A$ of $X$. Then $A$ is dense if and only if for every point $x$ of $X$, there exists a sequence $s_{2}$ of $X$ such that $\operatorname{rng} s_{2} \subseteq A$ and $s_{2}$ is convergent and $\lim s_{2}=x$. The theorem is a consequence of $(6)$.

(15) Let us consider a real normed space $X$, a subset $Y$ of $X$, and a subset $Z$ of LinearTopSpaceNorm $X$. If $Y=Z$, then $Y$ is dense iff $Z$ is dense. The theorem is a consequence of (3).

(16) Let us consider a real normed space $X$ and subsets $R, S$ of $X$. If $R$ is dense and $R \subseteq S$, then $S$ is dense. The theorem is a consequence of (15).

(17) Let us consider a real normed space $X$ and a subset $R$ of $X$. Then $R$ is dense if and only if for every subset $S$ of $X$ such that $S \neq \emptyset$ and $S$ is open holds $R$ meets $S$.

Proof: Reconsider $R_{1}=R$ as a subset of LinearTopSpaceNorm $X$. For every subset $S_{1}$ of LinearTopSpaceNorm $X$ such that $S_{1} \neq \emptyset$ and $S_{1}$ is open holds $R_{1}$ meets $S_{1}$ by [9, (33)].

Let us consider a real normed space $X$ and subsets $R, S$ of $X$. Now we state the propositions:

(18) If $R$ is dense and $S$ is open, then $\bar{S}=\overline{S \cap R}$. The theorem is a consequence of (3) and (15).

(19) If $R$ is dense and $S$ is dense and open, then $R \cap S$ is dense. The theorem is a consequence of (15).

(20) Let us consider a real normed space $X$ and a subset $A$ of $X$. If $A$ is dense, then $A$ is not empty. The theorem is a consequence of (17).

\section{Separability}

Let $X$ be a real normed space. We say that $X$ is separable if and only if (Def. 3) LinearTopSpaceNorm $X$ is separable.

(21) Let us consider a real normed space $X$. Then $X$ is separable if and only if there exists a sequence $s_{2}$ of $X$ such that $\operatorname{rng} s_{2}$ is dense. The theorem is a consequence of (15) and (20).

\section{Sequence And Convergence}

(22) Let us consider real numbers $x, y, z$. Suppose $0 \leqslant y$ and for every real number $e$ such that $0<e$ holds $x \leqslant z+y \cdot e$. Then $x \leqslant z$.

(23) Let us consider a real normed space $X$, a point $x$ of $X$, and a sequence $s_{2}$ of $X$. Suppose for every natural number $n, s_{2}(n)=x$. Then 
(i) $s_{2}$ is convergent, and

(ii) $\lim s_{2}=x$.

(24) Let us consider a real normed space $X$ and a point $x$ of $X$. Then $\{x\}$ is closed.

Proof: For every sequence $s_{1}$ of $X$ such that $\operatorname{rng} s_{1} \subseteq\{x\}$ and $s_{1}$ is convergent holds $\lim s_{1} \in\{x\}$ by [5, (4)], (23).

(25) Let us consider a real normed space $X$, a subset $Y$ of $X$, and a vector $v$ of $X$. Suppose $Y$ is closed and for every real number $e$ such that $0<e$ there exists a vector $w$ of $X$ such that $w \in Y$ and $\|v-w\| \leqslant e$. Then $v \in Y$.

\section{Subspace}

Now we state the propositions:

(26) Let us consider a real normed space $V$ and a subreal normal space $W$ of $V$. Suppose the carrier of $W=$ the carrier of $V$. Then the normed structure of $W=$ the normed structure of $V$.

(27) Let us consider a real normed space $V$. Then every subreal normal space of $V$ is a subspace of $V$.

(28) Let us consider a real normed space $V$, a subreal normal space $V_{1}$ of $V$, points $x, y$ of $V$, points $x_{1}, y_{1}$ of $V_{1}$, and a real number $a$. Suppose $x=x_{1}$ and $y=y_{1}$. Then

(i) $\|x\|=\left\|x_{1}\right\|$, and

(ii) $x+y=x_{1}+y_{1}$, and

(iii) $a \cdot x=a \cdot x_{1}$.

(29) Let us consider a real normed space $V$, a subreal normal space $V_{1}$ of $V$, and a subset $S$ of $V$. Suppose $S=$ the carrier of $V_{1}$. Then $S$ is linearly closed. The theorem is a consequence of (28).

Let $X$ be a real normed space and $X_{1}$ be a set. Assume $X_{1} \subseteq$ the carrier of $X$. The norm of $X_{1}$ induced by $X$ yielding a function from $X_{1}$ into $\mathbb{R}$ is defined by the term

(Def. 4) (the norm of $X) \uparrow X_{1}$.

Let $V$ be a real normed space and $V_{1}$ be a subset of $V$. The functor $\operatorname{NLin}\left(V_{1}\right)$ yielding a non empty normed structure is defined by the term

(Def. 5) 〈the carrier of $\operatorname{Lin}\left(V_{1}\right), 0_{\operatorname{Lin}\left(V_{1}\right)}$, the addition of $\operatorname{Lin}\left(V_{1}\right)$, the external multiplication of $\operatorname{Lin}\left(V_{1}\right)$, the norm of the carrier of $\operatorname{Lin}\left(V_{1}\right)$ induced by $\left.V\right\rangle$.

Now we state the proposition: 
(30) Let us consider a real normed space $V$ and a subset $V_{1}$ of $V$. Then $\operatorname{NLin}\left(V_{1}\right)$ is a subreal normal space of $V$.

Let $V$ be a real normed space and $V_{1}$ be a subset of $V$. Let us observe that the functor $\operatorname{NLin}\left(V_{1}\right)$ yields a subreal normal space of $V$. Now we state the propositions:

(31) Let us consider a real linear space $V$ and a subset $V_{1}$ of $V$. Suppose $V_{1} \neq \emptyset$ and $V_{1}$ is linearly closed. Then the carrier of $\operatorname{Lin}\left(V_{1}\right)=V_{1}$.

(32) Let us consider a real normed space $V$, a subreal normal space $W$ of $V$, and a subset $V_{1}$ of $V$. Suppose the carrier of $W=V_{1}$. Then $\operatorname{NLin}\left(V_{1}\right)=$ the normed structure of $W$. The theorem is a consequence of (31) and (29).

\section{Linear FunCtions}

Now we state the proposition:

(33) Let us consider real linear spaces $X, Y$ and a function $f$ from $X$ into $Y$. If $f$ is homogeneous, then $f^{-1}\left(\left\{0_{Y}\right\}\right)$ is not empty.

Let $X, Y$ be real linear spaces and $f$ be a linear operator from $X$ into $Y$. One can verify that $f^{-1}\left(\left\{0_{Y}\right\}\right)$ is non empty.

Let us consider real linear spaces $X, Y$ and a function $f$ from $X$ into $Y$.

Let us assume that $f$ is additive and homogeneous. Now we state the propositions:

(34) $f^{-1}\left(\left\{0_{Y}\right\}\right)$ is linearly closed.

Proof: Set $X_{1}=f^{-1}\left(\left\{0_{Y}\right\}\right)$. For every points $v, u$ of $X$ such that $v$, $u \in X_{1}$ holds $v+u \in X_{1}$ by [5, (38)], [27, (4)]. For every real number $r$ and for every point $v$ of $X$ such that $v \in X_{1}$ holds $r \cdot v \in X_{1}$ by [5, (38)], [27, (10)].

(35) $\operatorname{rng} f$ is linearly closed.

Proof: Set $Y_{1}=\operatorname{rng} f$. For every points $v, u$ of $Y$ such that $v, u \in Y_{1}$ holds $v+u \in Y_{1}$ by [5. (113), (4)]. For every real number $r$ and for every point $v$ of $Y$ such that $v \in Y_{1}$ holds $r \cdot v \in Y_{1}$ by [5, (113), (4)].

Let $X, Y$ be real linear spaces and $f$ be a linear operator from $X$ into $Y$. The functor Ker $f$ yielding a subspace of $X$ is defined by the term

(Def. 6) $\operatorname{Lin}\left(f^{-1}\left(\left\{0_{Y}\right\}\right)\right)$.

Let $X, Y$ be real normed spaces. The functor NKer $f$ yielding a subreal normal space of $X$ is defined by the term

(Def. 7) $\quad \mathrm{NLin}\left(\left(f^{-1}\left(\left\{0_{Y}\right\}\right)\right)\right)$. 
Let $X, Y$ be real linear spaces. The functor $\Im(f)$ yielding a subspace of $Y$ is defined by the term

(Def. 8) $\operatorname{Lin}(\operatorname{rng} f)$.

Let $X, Y$ be real normed spaces. The functor $\Im(f)$ yielding a subreal normal space of $Y$ is defined by the term

(Def. 9) $\quad \mathrm{NLin}(\operatorname{rng} f)$.

Let $X, Y$ be real linear spaces and $L$ be a linear operator from $X$ into $Y$. We say that $L$ is isomorphism if and only if

(Def. 10) $L$ is one-to-one and onto.

One can check that every linear operator from $X$ into $Y$ which is isomorphism is also one-to-one and onto and every linear operator from $X$ into $Y$ which is one-to-one and onto is also isomorphism.

Now we state the proposition:

(36) Let us consider real linear spaces $X, Y$ and a linear operator $L$ from $X$ into $Y$. Suppose $L$ is isomorphism. Then there exists a linear operator $K$ from $Y$ into $X$ such that

(i) $K=L^{-1}$, and

(ii) $K$ is isomorphism.

Proof: Reconsider $K=L^{-1}$ as a function from $Y$ into $X . K$ is additive by [5, (113)], [4, (34)]. $K$ is homogeneous by [5, (113)], [4, (34)].

Let $X, Y$ be real normed spaces and $L$ be a linear operator from $X$ into $Y$. We say that $L$ is isomorphism if and only if

(Def. 11) $L$ is one-to-one and onto and for every point $x$ of $X,\|x\|=\|L(x)\|$.

Let us note that every linear operator from $X$ into $Y$ which is isomorphism is also one-to-one and onto.

Now we state the propositions:

(37) Let us consider real normed spaces $X, Y$ and a linear operator $L$ from $X$ into $Y$. Suppose $L$ is isomorphism. Then there exists a Lipschitzian linear operator $K$ from $Y$ into $X$ such that

(i) $K=L^{-1}$, and

(ii) $K$ is isomorphism.

Proof: Reconsider $K=L^{-1}$ as a function from $Y$ into $X . K$ is additive by [5, (113)], [4, (34)]. $K$ is homogeneous by [5, (113)], [4, (34)]. For every point $y$ of $Y,\|y\|=\|K(y)\|$ by [5, (113)], [4, (34)].

(38) Let us consider real normed spaces $X, Y$, a Lipschitzian linear operator $L$ from $X$ into $Y$, and a sequence $s_{2}$ of $X$. Suppose $s_{2}$ is convergent. Then

(i) $L \cdot s_{2}$ is convergent, and 
(ii) $\lim \left(L \cdot s_{2}\right)=L\left(\lim s_{2}\right)$.

(39) Let us consider real normed spaces $X, Y$, a function $L$ from $X$ into $Y$, and a point $w$ of $Y$. Suppose $L$ is continuous on the carrier of $X$. Then $L^{-1}(\{w\})$ is closed.

Proof: For every sequence $s_{2}$ of $X$ such that $\operatorname{rng} s_{2} \subseteq L^{-1}(\{w\})$ and $s_{2}$ is convergent holds $\lim s_{2} \in L^{-1}(\{w\})$ by [15, (18)], [5, (4), (38), (115)].

(40) Let us consider real normed spaces $X, Y$ and a Lipschitzian linear operator $L$ from $X$ into $Y$. Then

(i) the carrier of $\operatorname{Ker} L=L^{-1}\left(\left\{0_{Y}\right\}\right)$, and

(ii) $L^{-1}\left(\left\{0_{Y}\right\}\right)$ is closed.

Let us consider real normed spaces $X, Y$, a Lipschitzian linear operator $L$ from $X$ into $Y$, and a sequence $s_{2}$ of $X$.

Let us assume that $L$ is isomorphism. Now we state the propositions:

(41) $s_{2}$ is convergent if and only if $L \cdot s_{2}$ is convergent.

Proof: Set $L_{3}=L \cdot s_{2}$. Consider $K$ being a Lipschitzian linear operator from $Y$ into $X$ such that $K=L^{-1}$ and $K$ is isomorphism. For every element $n$ of $\mathbb{N},\left(K \cdot L_{3}\right)(n)=s_{2}(n)$ by [4, (13), (34)].

(42) If $s_{2}$ is Cauchy sequence by norm, then $L \cdot s_{2}$ is Cauchy sequence by norm.

Proof: Set $L_{3}=L \cdot s_{2}$. For every real number $r$ such that $r>0$ there exists a natural number $k$ such that for every natural numbers $n, m$ such that $n \geqslant k$ and $m \geqslant k$ holds $\left\|L_{3}(n)-L_{3}(m)\right\|<r$ by [22, (8)], [4, (13)], [27, (16)].

(43) $s_{2}$ is Cauchy sequence by norm if and only if $L \cdot s_{2}$ is Cauchy sequence by norm.

Proof: Set $L_{3}=L \cdot s_{2}$. Consider $K$ being a Lipschitzian linear operator from $Y$ into $X$ such that $K=L^{-1}$ and $K$ is isomorphism. For every element $n$ of $\mathbb{N},\left(K \cdot L_{3}\right)(n)=s_{2}(n)$ by [4, (13), (34)].

Now we state the propositions:

(44) Let us consider real normed spaces $X, Y$. Suppose there exists a Lipschitzian linear operator $L$ from $X$ into $Y$ such that $L$ is isomorphism. Then $X$ is complete if and only if $Y$ is complete. The theorem is a consequence of (37), (43), and (41).

(45) Let us consider real normed spaces $X, Y$, a Lipschitzian linear operator $L$ from $X$ into $Y$, a subset $V$ of $X$, and a subset $W$ of $Y$. Suppose $L$ is isomorphism and $W=L^{\circ} V$. Then $V$ is closed if and only if $W$ is closed. The theorem is a consequence of (37). 
(46) Let us consider real normed spaces $X, Y$ and a linear operator $L$ from $X$ into $Y$. Suppose $L$ is onto. Then $\Im(L)=$ the normed structure of $Y$. The theorem is a consequence of (31), (35), and (26).

\section{Banach SPACE}

Now we state the propositions:

(47) Let us consider a real Banach space $V$ and a subreal normal space $V_{1}$ of $V$. Suppose there exists a subset $C_{2}$ of $V$ such that $C_{2}=$ the carrier of $V_{1}$ and $C_{2}$ is closed. Then $V_{1}$ is a real Banach space.

Proof: For every sequence $s_{2}$ of $V_{1}$ such that $s_{2}$ is Cauchy sequence by norm holds $s_{2}$ is convergent by [5, (7)], [22, (8)], [27, (16)], (28).

(48) Let us consider a real normed space $V$, a subreal normal space $V_{1}$ of $V$, and a subset $C_{2}$ of $V$. Suppose $V_{1}$ is complete and $C_{2}=$ the carrier of $V_{1}$. Then $C_{2}$ is closed.

Proof: For every sequence $s_{1}$ of $V$ such that $\operatorname{rng} s_{1} \subseteq C_{2}$ and $s_{1}$ is convergent holds $\lim s_{1} \in C_{2}$ by [5, (6)], [21, (4)], [27, (16)], (28).

(49) Let us consider a real Banach space $X$ and a non empty subset $M$ of $X$. Suppose $M$ is linearly closed and closed. Then $\operatorname{NLin}(M)$ is a real Banach space. The theorem is a consequence of (31) and (47).

\section{Quotient Vector Space}

Let $X$ be a real linear space and $Y$ be a subspace of $X$. Observe that the functor RLSp2RVSp $Y$ yields a subspace of RLSp2RVSp $X$. The functor $X / Y$ yielding a real linear space is defined by the term

(Def. 12) RVSp2RLSp $\left({ }^{\operatorname{RLSp} 2 \operatorname{RVSp} X} / \operatorname{RLSp2RVSp} Y\right)$.

Now we state the propositions:

(50) Let us consider a real linear space $X$, an element $v$ of $X$, a real number $a$, an element $v_{1}$ of $\operatorname{RLSp} 2 \operatorname{RVSp} X$, and an element $a_{1}$ of $\mathbb{R}_{\mathrm{F}}$. If $v=v_{1}$ and $a=a_{1}$, then $a \cdot v=a_{1} \cdot v_{1}$.

(51) Let us consider a vector space $X$ over $\mathbb{R}_{\mathrm{F}}$, an element $v$ of $X$, an element $a$ of $\mathbb{R}_{\mathrm{F}}$, an element $v_{1}$ of RVSp2RLSp $X$, and a real number $a_{1}$. If $v=v_{1}$ and $a=a_{1}$, then $a \cdot v=a_{1} \cdot v_{1}$.

(52) Let us consider a real linear space $X$, a subspace $Y$ of $X$, an element $v$ of $X$, and an element $v_{1}$ of RLSp2RVSp $X$. If $v=v_{1}$, then $v+Y=$ $v_{1}+\operatorname{RLSp} 2 \operatorname{RVSp} Y$. 
(53) Let us consider a real linear space $X$, a subspace $Y$ of $X$, and an object $x$. Then $x$ is a coset of $Y$ if and only if $x$ is a coset of RLSp2RVSp $Y$. The theorem is a consequence of (52).

Let $X$ be a real linear space and $Y$ be a subspace of $X$. The functor $\operatorname{CosetSet}(X, Y)$ yielding a non empty family of subsets of $X$ is defined by the term

(Def. 13) the set of all $A$ where $A$ is a coset of $Y$.

Let $V$ be a real linear space and $W$ be a subspace of $V$. The functor zeroCoset $(V, W)$ yielding an element of $\operatorname{CosetSet}(V, W)$ is defined by the term

(Def. 14) the carrier of $W$.

Now we state the propositions:

(54) Let us consider a real linear space $X$ and a subspace $Y$ of $X$. Then $\operatorname{CosetSet}(X, Y)=\operatorname{CosetSet}(\operatorname{RLSp} 2 \operatorname{RVSp} X, \operatorname{RLSp} 2 \mathrm{RVSp} Y)$. The theorem is a consequence of (53).

(55) Let us consider a real linear space $V$ and a subspace $W$ of $V$. Then the carrier of $V / W=\operatorname{CosetSet}(V, W)$. The theorem is a consequence of (54).

(56) Let us consider a real linear space $V$, a subspace $W$ of $V$, and an object $x$. Then $x$ is a point of $V / W$ if and only if there exists a point $v$ of $V$ such that $x=v+W$. The theorem is a consequence of (55).

(57) Let us consider a real linear space $V$ and a subspace $W$ of $V$. Then $0_{V / W}=\operatorname{zeroCoset}(V, W)$.

Let us consider a real linear space $V$, a subspace $W$ of $V$, a vector $A$ of $V / W$, a vector $v$ of $V$, and a real number $a$.

Let us assume that $A=v+W$. Now we state the propositions:

(58) $a \cdot A=a \cdot v+W$. The theorem is a consequence of (52).

(59) $-A=-v+W$. The theorem is a consequence of (58).

Let us consider a real linear space $V$, a subspace $W$ of $V$, vectors $A_{1}, A_{2}$ of $V / W$, and vectors $v_{1}, v_{2}$ of $V$.

Let us assume that $A_{1}=v_{1}+W$ and $A_{2}=v_{2}+W$. Now we state the propositions:

(60) $A_{1}+A_{2}=v_{1}+v_{2}+W$. The theorem is a consequence of (52).

(61) $A_{1}-A_{2}=v_{1}-v_{2}+W$. The theorem is a consequence of (59) and (60).

Let us consider a real linear space $V$ and a subspace $W$ of $V$. Now we state the propositions:

(62) (i) $0_{V} / W=$ the carrier of $W$, and

(ii) $0_{V / W}=0_{V}+W$. 
The theorem is a consequence of (57).

(63) There exists a linear operator $Q_{2}$ from $V$ into $V / W$ such that

(i) $Q_{2}$ is onto, and

(ii) for every vector $v$ of $V, Q_{2}(v)=v+W$.

Proof: Define $\mathcal{P}$ [vector of $V$, object] $\equiv \$_{2}=\$_{1}+W$. For every element $x$ of the carrier of $V$, there exists an element $y$ of the carrier of $V / W$ such that $\mathcal{P}[x, y]$. Consider $Q_{2}$ being a function from the carrier of $V$ into $V / W$ such that for every element $x$ of $V, \mathcal{P}\left[x, Q_{2}(x)\right]$ from [5, Sch. 3]. For every elements $v, w$ of $V, Q_{2}(v+w)=Q_{2}(v)+Q_{2}(w)$. For every vector $v$ of $V$ and for every real number $r, Q_{2}(r \cdot v)=r \cdot Q_{2}(v)$. For every object $v$ such that $v \in$ the carrier of $V / W$ there exists an object $s$ such that $s \in$ the carrier of $V$ and $v=Q_{2}(s)$.

Let $V$ be a real linear space and $W$ be a subspace of $V$. The surjection induced by $(V, W)$ yielding a linear operator from $V$ into $V / W$ is defined by

(Def. 15) it is onto and for every vector $v$ of $V$, it $(v)=v+W$.

Now we state the proposition:

(64) Let us consider real linear spaces $V, W$ and a linear operator $L$ from $V$ into $W$. Then there exists a linear operator $Q_{2}$ from $V / \operatorname{Ker} L$ into $\Im(L)$ such that

(i) $Q_{2}$ is isomorphism, and

(ii) for every point $z$ of $V / \operatorname{Ker} L$ and for every vector $v$ of $V$ such that $z=v+\operatorname{Ker} L$ holds $Q_{2}(z)=L(v)$.

Proof: The carrier of $\Im(L)=\operatorname{rng} L$. The carrier of $\operatorname{Ker} L=L^{-1}\left(\left\{0_{W}\right\}\right)$. Define $\mathcal{P}$ [object, object] $\equiv$ there exists a vector $v$ of $V$ such that $\$_{1}=$ $v+\operatorname{Ker} L$ and $\$_{2}=L(v)$. For every element $x$ of the carrier of $V / \operatorname{Ker} L$, there exists an element $y$ of the carrier of $\Im(L)$ such that $\mathcal{P}[x, y]$ by $(56)$, [5. (4)]. Consider $Q_{2}$ being a function from the carrier of $V / \operatorname{Ker} L$ into the carrier of $\Im(L)$ such that for every element $x$ of $V / \operatorname{Ker} L, \mathcal{P}\left[x, Q_{2}(x)\right]$ from [5, Sch. 3]. For every point $z$ of $V / \operatorname{Ker} L$ and for every vector $v$ of $V$ such that $z=v+\operatorname{Ker} L$ holds $Q_{2}(z)=L(v)$ by [26, (54), (63)], 27, (28), (15), (4)]. For every objects $x_{1}, x_{2}$ such that $x_{1}, x_{2} \in$ the carrier of $V / \operatorname{Ker} L$ and $Q_{2}\left(x_{1}\right)=Q_{2}\left(x_{2}\right)$ holds $x_{1}=x_{2}$ by [27, (16), (15)], [5, (38)], [27, (29), (13)]. For every object $v$ such that $v \in$ the carrier of $\Im(L)$ there exists an object $s$ such that $s \in$ the carrier of $V / \operatorname{Ker} L$ and $v=Q_{2}(s)$ by (35), (31), 5. (11)], (56). For every elements $v, w$ of $V / \operatorname{Ker} L, Q_{2}(v+w)=Q_{2}(v)+Q_{2}(w)$ by (56), (60), [26, (13)]. For every vector $v$ of $V / \operatorname{Ker} L$ and for every real number $r, Q_{2}(r \cdot v)=r \cdot Q_{2}(v)$ by $(56),(58)$, [26, (14)]. 
Let $V, W$ be real linear spaces and $L$ be a linear operator from $V$ into $W$. The bijection induced by $(V, W, L)$ yielding a linear operator from ${ }^{V} / \operatorname{Ker} L$ into $\Im(L)$ is defined by

(Def. 16) it is isomorphism and for every point $z$ of $V / \operatorname{Ker} L$ and for every vector $v$ of $V$ such that $z=v+\operatorname{Ker} L$ holds $i t(z)=L(v)$.

Now we state the proposition:

(65) Let us consider real linear spaces $V, W$ and a linear operator $L$ from $V$ into $W$. Then $L=$ (the bijection induced by $(V, W, L)) \cdot($ the surjection induced by $(V$, $\operatorname{Ker} L))$. The theorem is a consequence of (56).

Let $V$ be a real normed space, $W$ be a subspace of $V$, and $v$ be a vector of $V$. The functor $\operatorname{NormVSets}(V, W, v)$ yielding a non empty subset of $\mathbb{R}$ is defined by the term

(Def. 17) $\{\|x\|$, where $x$ is a vector of $V: x \in v+W\}$.

Let us observe that $\operatorname{NormVSets}(V, W, v)$ is non empty and lower bounded. Now we state the proposition:

(66) Let us consider a real normed space $V$, a subspace $W$ of $V$, and a vector $v$ of $V$. Then $0 \leqslant \inf \operatorname{NormVSets}(V, W, v) \leqslant\|v\|$.

Let $V$ be a real normed space and $W$ be a subspace of $V$. The functor $\operatorname{Norm} \operatorname{Coset}(V, W)$ yielding a function from $\operatorname{CosetSet}(V, W)$ into $\mathbb{R}$ is defined by

(Def. 18) for every element $A$ of $\operatorname{CosetSet}(V, W)$ and for every vector $v$ of $V$ such that $A=v+W$ holds it $(A)=\inf \operatorname{Norm} \operatorname{VSets}(V, W, v)$.

Let $X$ be a real normed space and $Y$ be a subspace of $X$. Assume there exists a subset $C_{3}$ of $X$ such that $C_{3}=$ the carrier of $Y$ and $C_{3}$ is closed. The functor $\operatorname{NVectQuot}(X, Y)$ yielding a strict real normed space is defined by

(Def. 19) the RLS structure of $i t=X / Y$ and the norm of $i t=\operatorname{NormCoset}(X, Y)$.

Now we state the proposition:

(67) Let us consider real normed spaces $V, W$ and a Lipschitzian linear operator $L$ from $V$ into $W$. Then there exists a Lipschitzian linear operator $Q_{2}$ from $\operatorname{NVectQuot}(V,(\operatorname{Ker} L))$ into $\Im(L)$ and there exists a point $P_{3}$ of the real norm space of bounded linear operators from $\operatorname{NVectQuot}(V,(\operatorname{Ker} L))$ into $\Im(L)$ and there exists a point $P_{2}$ of the real norm space of bounded linear operators from $V$ into $W$ such that $Q_{2}$ is onto and one-to-one and $L=P_{2}$ and $Q_{2}=P_{3}$ and $\left\|P_{2}\right\|=\left\|P_{3}\right\|$ and for every point $z$ of $\operatorname{NVectQuot}(V,(\operatorname{Ker} L))$ and for every vector $v$ of $V$ such that $z=v+\operatorname{Ker} L$ holds $Q_{2}(z)=L(v)$.

Proof: the carrier of $\operatorname{Ker} L=L^{-1}\left(\left\{0_{W}\right\}\right)$ and $L^{-1}\left(\left\{0_{W}\right\}\right)$ is closed. Reconsider $V_{1}=V$ as a real linear space. Reconsider $W_{1}=W$ as a real linear space. Reconsider $L_{1}=L$ as a linear operator from $V_{1}$ into $W_{1}$. 
The carrier of $V / \operatorname{Ker} L=\operatorname{Coset} \operatorname{Set}(V$, Ker $L)$. Consider $Q_{3}$ being a linear operator from $V_{1} / \operatorname{Ker} L_{1}$ into $\Im\left(L_{1}\right)$ such that $Q_{3}$ is isomorphism and for every point $z$ of $V_{1} / \operatorname{Ker} L_{1}$ and for every vector $v$ of $V_{1}$ such that $z=v+\operatorname{Ker} L_{1}$ holds $Q_{3}(z)=L_{1}(v)$. Reconsider $Q_{2}=Q_{3}$ as a function from $\operatorname{NVectQuot}(V,(\operatorname{Ker} L))$ into $\Im(L)$. For every elements $v, w$ of $\operatorname{NVectQuot}(V,(\operatorname{Ker} L)), Q_{2}(v+w)=Q_{2}(v)+Q_{2}(w)$. For every vector $v$ of $\operatorname{NVectQuot}(V,(\operatorname{Ker} L))$ and for every real number $r, Q_{2}(r \cdot v)=r \cdot Q_{2}(v)$. Reconsider $P_{2}=L$ as a point of the real norm space of bounded linear operators from $V$ into $W$. For every point $v$ of $\operatorname{NVectQuot}(V,(\operatorname{Ker} L))$, $\left\|Q_{2}(v)\right\| \leqslant\left\|P_{2}\right\| \cdot\|v\|$ by (56), [20, (31)], [24, (7)], (28). Reconsider $P_{3}=$ $Q_{2}$ as a point of the real norm space of bounded linear operators from $\operatorname{NVectQuot}(V,(\operatorname{Ker} L))$ into $\Im(L)$. $\left\|P_{2}\right\| \leqslant\left\|P_{3}\right\|$.

\section{Closure}

Let $X$ be a real normed space and $Y$ be a subset of $X$. The functor $\operatorname{ClNLin}(Y)$ yielding a non empty normed structure is defined by

(Def. 20) there exists a subset $Z$ of $X$ such that $Z=$ the carrier of $\operatorname{Lin}(Y)$ and it $=\langle\bar{Z}, \operatorname{Zero}(\bar{Z}, X), \operatorname{Add}(\bar{Z}, X), \operatorname{Mult}(\bar{Z}, X)$, the norm of $\bar{Z}$ induced by $X\rangle$.

Now we state the propositions:

(68) Let us consider a real normed space $X$, a subset $V_{1}$ of $X$, and a subset $C_{1}$ of $X$. Suppose $C_{1}=$ the carrier of $\operatorname{ClNLin}\left(V_{1}\right)$. Then $\left\langle C_{1}, \operatorname{Zero}\left(C_{1}, X\right)\right.$, $\left.\operatorname{Add}\left(C_{1}, X\right), \operatorname{Mult}\left(C_{1}, X\right)\right\rangle$ is a subspace of $X$. The theorem is a consequence of (13).

(69) Let us consider a real normed space $X$, a subset $Y$ of $X$, points $f, g$ of $\operatorname{ClNLin}(Y)$, and a real number $a$. Then

(i) $\|f\|=0$ iff $f=0_{\operatorname{ClNLin}(Y)}$, and

(ii) $\|a \cdot f\|=|a| \cdot\|f\|$, and

(iii) $\|f+g\| \leqslant\|f\|+\|g\|$.

The theorem is a consequence of (13).

Let $X$ be a real normed space and $Y$ be a subset of $X$. Let us observe that $\operatorname{ClNLin}(Y)$ is reflexive, discernible, and real normed space-like.

Now we state the proposition:

(70) Let us consider a real normed space $V$ and a subset $V_{1}$ of $V$. Then $\operatorname{ClNLin}\left(V_{1}\right)$ is a real normed space. The theorem is a consequence of $(68)$. 
Let $X$ be a real normed space and $Y$ be a subset of $X$. Let us observe that $\operatorname{ClNLin}(Y)$ is reflexive, discernible, real normed space-like, vector distributive, scalar distributive, scalar associative, scalar unital, Abelian, add-associative, right zeroed, and right complementable.

Now we state the proposition:

(71) Let us consider a real normed space $V$ and a subset $V_{1}$ of $V$. Then $\operatorname{ClNLin}\left(V_{1}\right)$ is a subreal normal space of $V$. The theorem is a consequence of (13).

Let $V$ be a real normed space and $V_{1}$ be a subset of $V$. One can verify that the functor ClNLin $\left(V_{1}\right)$ yields a subreal normal space of $V$.

\section{REFERENCES}

[1] Grzegorz Bancerek. The ordinal numbers. Formalized Mathematics, 1(1):91-96, 1990.

[2] Nicolas Bourbaki, H.G. Eggleston, and S. Madan. Elements of mathematics: Topological vector spaces. Springer-Verlag, 1987.

[3] Czesław Byliński. The complex numbers. Formalized Mathematics, 1(3):507-513, 1990.

[4] Czesław Byliński. Functions and their basic properties Formalized Mathematics, 1(1): 55-65, 1990.

[5] Czesław Byliński. Functions from a set to a set Formalized Mathematics, 1(1):153-164, 1990.

[6] Czesław Byliński. Partial functions. Formalized Mathematics, 1(2):357-367, 1990.

[7] Czesław Byliński. Some basic properties of sets Formalized Mathematics, 1(1):47-53, 1990.

[8] Noboru Endou, Yasumasa Suzuki, and Yasunari Shidama. Real linear space of real sequences Formalized Mathematics, 11(3):249-253, 2003.

[9] Noboru Endou, Yasunari Shidama, and Katsumasa Okamura. Baire's category theorem and some spaces generated from real normed space. Formalized Mathematics, 14(4): 213-219, 2006. doi $10.2478 /$ v10037-006-0024-x.

[10] Adam Grabowski. On the boundary and derivative of a set Formalized Mathematics, 13 (1):139-146, 2005.

[11] Jarosław Kotowicz. Convergent real sequences. Upper and lower bound of sets of real numbers Formalized Mathematıcs, 1(3):477-481, 1990.

[12] Jarosław Kotowicz. Quotient vector spaces and functionals. Formalized Mathematics, 11 (1):59-68, 2003.

[13] Eugeniusz Kusak, Wojciech Leończuk, and Michał Muzalewski. Abelian groups, fields and vector spaces. Formalized Mathematics, 1(2):335-342, 1990.

[14] Keiko Narita, Noboru Endou, and Yasunari Shidama. Dual spaces and Hahn-Banach theorem. Formalized Mathematics, 22(1):69-77, 2014. doi 10.2478/forma-2014-0007.

[15] Takava Nishivama, Keiji Ohkubo, and Yasunari Shidama. The continuous functions on normed linear spaces Formalized Mathematics, 12(3):269-275, 2004.

[16] Beata Padlewska. Families of sets Formalized Mathematics, 1(1):147-152, 1990.

[17] Beata Padlewska and Agata Darmochwał. Topological spaces and continuous functions. Formalized Mathematics, 1(1):223-230, 1990.

[18] Jan Popiołek. Real normed space Formalized Mathematics, 2(1):111-115, 1991.

[19] Walter Rudin. Functional Analysis. New York, McGraw-Hill, 2nd edition, 1991.

[20] Yasunari Shidama. Banach space of bounded linear operators Formalized Mathematics, 12(1):39-48, 2004.

[21] Yasunari Shidama. The series on Banach algebra. Formalized Mathematics, 12(2):131$138,2004$. 
[22] Yasumasa Suzuki, Noboru Endou, and Yasunari Shidama. Banach space of absolute summable real sequences Formalized Mathematics, 11(4):377-380, 2003.

[23] Andrzej Trybulec. Domains and their Cartesian products. Formalized Mathematics, 1(1): $115-122,1990$.

[24] Andrzej Trybulec. Binary operations applied to functions Formalized Mathematics, 1 (2):329-334, 1990.

[25] Andrzej Trybulec. On the sets inhabited by numbers. Formalized Mathematics, 11(4): 341-347, 2003.

[26] Wojciech A. Trybulec. Subspaces and cosets of subspaces in real linear space Formalized Mathematics, 1(2):297-301, 1990.

[27] Wojciech A. Trybulec. Vectors in real linear space. Formalized Mathematics, 1(2):291-296, 1990.

[28] Wojciech A. Trybulec. Basis of real linear space. Formalized Mathematics, 1(5):847-850, 1990.

[29] Wojciech A. Trybulec. Subspaces and cosets of subspaces in vector space. Formalized Mathematics, 1(5):865-870, 1990.

[30] Zinaida Trybulec. Properties of subsets Formalized Mathematics, 1(1):67-71, 1990.

[31] Edmund Woronowicz. Relations and their basic properties Formalized Mathematics, 1 (1):73-83, 1990.

[32] Edmund Woronowicz. Relations defined on sets. Formalized Mathematics, 1(1):181-186, 1990.

[33] Mirosław Wysocki and Agata Darmochwał. Subsets of topological spaces. Formalized Mathematics, 1(1):231-237, 1990.

[34] Kosaku Yoshida. Functional Analysis. Springer, 1980.

Received September 15, 2014 